\title{
Universiteit
}

Leiden

The Netherlands

\section{The relationship between guilt feelings, conflicts with staff and satisfaction with care in relatives of nursing home residents with dementia: a longitudinal analysis \\ Gallego-Alberto, L.; Smaling, H.J.A.; Francke, A.L.; Brug, T. van de; Steen, J.T. van der}

\section{Citation}

Gallego-Alberto, L., Smaling, H. J. A., Francke, A. L., Brug, T. van de, \& Steen, J. T. van der. (2021). The relationship between guilt feelings, conflicts with staff and satisfaction with care in relatives of nursing home residents with dementia: a longitudinal analysis. Dementia, 21(1).

doi:10.1177/14713012211024015

Version: $\quad$ Publisher's Version

License: $\quad$ Creative Commons CC BY 4.0 license

Downloaded from: $\quad$ https://hdl.handle.net/1887/3276144

Note: To cite this publication please use the final published version (if applicable). 
Dementia

202I, Vol. 0(0) I-I6

(C) The Author(s) 2021

Article reuse guidelines:

sagepub.com/journals-permissions DOI: 10.1 I77/147/3012211024015

journals.sagepub.com/home/dem

(3)SAGE

\section{Laura Gallego-Alberto}

Faculty of Psychology, Autonomous University of Madrid, Department of Biological and Health Psychology, Madrid, Spain

\section{Hanneke JA Smaling}

Department of Public Health and Primary Care, Leiden University Medical Center, Leiden, The Netherlands; Department of Public and Occupational Health, Amsterdam University Medical Centers, Location VU University Medical Center, Amsterdam, The Netherlands

\section{Anneke L Francke}

Nivel - Netherlands Institute of Health Services Research in Utrecht, and VU University Medical Center, Amsterdam Public Health Research Institute, Amsterdam, The Netherlands

\section{Tim van de Brug}

Department of Epidemiology and Biostatistics, VU University Medical Center, Amsterdam University Medical Centers, Amsterdam, The Netherlands

\section{Jenny $T$ van der Steen \\ Department of Public Health and Primary Care, Leiden University Medical Center, Leiden, The Netherlands; Department of Primary and Community Care, Radboud university medical center, Nijmegen, The Netherlands; Department of Public and Occupational Health, Amsterdam University Medical Centers, VU University Medical Center, Amsterdam, The Netherlands}

\section{Corresponding author:}

Jenny T van der Steen, Department of Public Health and Primary Care, Leiden University Medical Center, Hippocratespad 2I, Leiden 2333, Zuid-Holland, The Netherlands.

Email: J.T.van_der_Steen@lumc.nl 


\title{
Karlijn J Joling
}

Department of General Practice and Elderly Care Medicine, Amsterdam

Public Health Research Institute, Vrije Universiteit Amsterdam,

Amsterdam, Netherlands

\begin{abstract}
Introduction: Family caregivers often experience guilt after nursing home placement. The aim of the present study was to describe family caregivers' guilt over time and assess the impact of conflicts with staff and satisfaction with care on guilt.
\end{abstract}

Method: Data of 222 family caregivers at three assessments during one-year follow-up were used. In addition to caregivers' guilt and the variables conflicts with staff and satisfaction with the care, potential confounders were measured: sociodemographic data, clinical characteristics of the person with dementia, and caregiver burden. Linear mixed model analyses were performed to examine the longitudinal relationships between variables.

Results: Guilt remained stable over time. Unadjusted models showed that conflicts with staff were positively associated with guilt $(\beta=0.1 \mathrm{I} ; p<0.00 \mathrm{I} ; 95 \% \mathrm{Cl}: 0.06$ to 0.16$)$ and satisfaction with care showed a negative association with guilt $(\beta=-0.10 ; p<0.05 ; 95 \% \mathrm{Cl}:-0.18$ to $-0.0 \mathrm{I})$. After adjusting for the confounders, only the positive association of guilt with conflicts with staff was similar as in the unadjusted analysis $(\beta=0.1 \mathrm{I} ; p<0.00 \mathrm{I} ; 95 \% \mathrm{Cl}$ : 0.05 to 0.16$)$, whereas satisfaction with care was not significantly associated with guilt in the adjusted analyses $(\beta=-0.07 ; p=0.10 ; 95 \%$ $\mathrm{Cl}:-0.16$ to $0.0 \mathrm{I})$.

Discussion: More conflicts with staff are associated with stronger guilt feelings. Guilt feelings are experienced by caregivers even after the admission of the person with dementia, and they remain stable over time. Further studies should focus on how to address guilt in family caregivers of people with dementia living in nursing homes.

\section{Keywords}

family caregivers, nursing home, guilt, conflicts with staff, satisfaction with care, burden

\section{Introduction}

Over the last decades, dementia caregiving has been linked to negative outcomes in family caregivers' physical and psychological health (Ma et al., 2018; Pinquart \& Sorensen, 2003). When the care recipients' disease progresses, the number and intensity of their demands increase and the nursing home placement of the person with dementia often becomes an inevitable decision for family caregivers at some point in time (Houttekier et al., 2010; Nikzad-Terhure et al., 2010; Toot et al., 2017). Although a nursing home admission could be a relief for family caregivers (Gaugler et al., 2009), several studies have shown that burden and emotional distress continue after institutionalization (Bern-Klug \& Forbes-Thompson, 2008; Hennings et al., 2013; Gaugler et al., 2007; Sury et al., 2013).

Guilt is a frequent emotion in family caregivers after nursing home admission of their relative (Bern-Klug, \& Forbes-Thompson, 2008; Church et al., 2016; Davis et al., 2019; Hennings et al., 2013). Guilt has been defined as "the dysphoric feeling associated with the recognition that one has violated a personally relevant moral or social standard" (Kugler \& Jones, 1992, p. 318). In the 
dementia family caregiving literature, guilt has been associated with higher levels of burden and poor mental health outcomes, such as depressive, anxious, and ambivalent symptoms (Feast et al., 2017; Losada et al., 2018). Although guilt can arise through the entire caregiving process, family caregivers usually report feelings of guilt when the person with dementia is admitted to or resides in a nursing home (e.g., Bern-Klug \& Forbes-Thompson, 2008; Church et al., 2016; Harstäde et al., 2013; Park, 2002; Ryan \& Scullion, 2000; Sury et al., 2013). Guilt may not only constitute a temporal emotional response to the admission, as Hennings et al. (2013) found it persistent in the four years following the admission, and even in some cases it still continued after the death of the relative (Harrop et al., 2016).

The majority of the studies are consistent in considering guilt feelings to be a consequence of nursing home admission, highlighting the role of negative self-evaluations referring to not doing enough as a caregiver and over abandoning or betraying their relative with dementia (Cronfalk et al., 2017; Graneheim et al., 2014; Høgsnes et al., 2014). In addition, higher perceived burden levels are associated with more feelings of guilt (Davis et al., 2019). Furthermore, the interaction and dynamics between the caregiver and the nursing home care setting and staff appear to play an important role in the guilt and distress experienced by family caregivers (Bern-Klug \& Forbes-Thompson, 2008; Givens et al., 2012; Park, 2002; Roberts \& Ishler, 2017; Sury et al., 2013).

As the caregiving role does not stop after nursing home placement, family caregivers must adapt their caring tasks and responsibilities (e.g., no leading role anymore with physical care such as bathing and adopt a role related to monitoring or assisting the staff). However, the involvement of family caregivers and their relationship with the nursing home staff may be challenging, and may lead to feelings of burden, stress, and negative emotions, such as anxiety or guilt for both families and staff (Church et al., 2016; Haesler et al., 2007; Gallego-Alberto et al., 2018; Roberts \& Ishler, 2017). The cross-sectional studies that have analyzed guilt experienced by the family caregivers found that higher levels of guilt are related to fewer family visits, lower levels of satisfaction with the care provided by the staff, and a higher frequency of conflicts with the staff (Bern-Klug \& ForbesThompson, 2008; Davis et al., 2019; Gladstone et al., 2006; Park, 2002). Recently, Davis et al. (2019) found that levels of guilt after admission showed significant positive associations with family caregivers' burden, depression, and conflicts with staff. Furthermore, guilt feelings were negatively associated with caregivers' general well-being.

Nevertheless, the few studies that addressed the relationship between guilt and family involvement (i.e., interactions with staff) or family satisfaction with the care offered in the nursing home are qualitative or cross-sectional. Hence, the present study aims to examine the longitudinal relationship between guilt feelings and conflicts with nursing home staff and satisfaction with the care offered by staff in a large sample of family caregivers of nursing home residents with dementia. In addition, we examine the impact of potential confounding factors, such as family caregiver burden, on this relationship.

\section{Method}

\section{Design and setting}

Data were derived from the Namaste Care Family trial (Smaling et al., 2018). The principal objective of the study was to evaluate the impact of the Namaste Care Family program on quality of life of residents with dementia and on positive caregiving experiences of their family caregivers. The Namaste Care Family intervention aims to increase the quality of life of people with advanced dementia by addressing any needs of residents in a person-centered way. 
Family caregivers who participated in the study were recruited through 19 nursing homes located in the Netherlands. The inclusion criteria for participation in the study were having a relative with dementia living in a nursing home; an expectation by the nursing home staff that the family caregiver or resident may benefit from the program; and to understand the Dutch language and willingness to fill in questionnaires. Before enrolling in the study, the family caregivers provided informed consent. The 19 participating nursing homes were matched on several criteria (i.e., rural vs urban area, etc.) in order to control for different aspects which may affect the intervention's effect. Once matched, the nursing homes were randomly assigned to either the intervention or control condition. Ten nursing homes participated in the intervention condition (Namaste Care Family intervention) and nine in the control condition (usual care). More details about the intervention, sample recruitment and procedures are available in the protocol article of the Namaste Care Family trial (Smaling et al., 2018). For the current study, to analyze the longitudinal relationship between guilt and conflicts with staff and satisfaction with care, data from the intervention and control conditions were combined as the intervention did not significantly change feelings of guilt. This research was supported by the the Netherlands Organisation for Health Research and Development (ZonMw) grant number 733,050,302, Fonds NutsOhra (FNO) grant number 1405-181, and University Network of the Care sector South Holland (UNC-ZH).

\section{Measures}

The family caregivers filled in questionnaires online or on paper at baseline and at three and 12 months after the baseline assessment.

\section{Outcome measure}

Guilt feelings. The primary outcome for the current analyses was feelings of guilt experienced by family caregivers over the 12-month follow-up period. Guilt feelings were measured with the subscale of "guilt from perceived failure in caregiving" of the Family Perceptions of Caregiving Role (FPCR; Maas et al., 2004). That subscale comprises five items (e.g., I feel guilty about my interactions with my relative). The original FPCR instrument has a seven-point agreement scale (with end points 1 = strongly disagree to $7=$ strongly agree). In the current study, we used the fivepoint agreement scale with verbal labels used by Zimmerman et al. (2013) (categories: 1 = strongly disagree; 2 = disagree; $3=$ neither disagree nor agree; $4=$ agree; and $5=$ strongly agree), who found that the FPCR with that modification was sensitive to change. Sum scores range from 5 to 25 , with higher scores indicating higher feelings of guilt. Cronbach's alpha for this scale was 0.75 at baseline, 0.77 at the second assessment, and 0.79 at the third assessment.

\section{Independent variables}

Conflicts with nursing home staff. To assess the family caregivers' perceptions of conflicts with care staff, the subscale "conflict with staff over caregiving" of the FPCR (Maas et al., 2004) was used. The subscale is composed of 10 items (e.g., Staff listen to my directions for my relative's care, but ignore them if they choose) with the Likert type-response, used by Zimmerman et al. (2013), ranging from 1 (strongly disagree) to 5 (strongly agree). Total scores range from 10 to 50 with higher scores indicating higher levels of conflicts between the caregiver and nursing home staff. Cronbach's alpha for this scale was 0.82 at baseline and 0.86 and 0.81 at the second and third assessments, respectively. 
Satisfaction with care. The caregiver's satisfaction with the care the resident received was assessed with the End-of-Life in Dementia-Satisfaction With Care scale (EOLD-SWC; Volicer et al., 2001). It comprises 10 items (e.g., I feel myself fully involved in all decisions) with a Likert type-response ranging from 1 (strongly disagree) to 4 (strongly agree). Total scores range from 10 to 40 , and higher scores indicate higher satisfaction with the care offered by the nursing home staff. Cronbach's alpha at the baseline assessment was 0.82 and 0.85 and 0.86 at the second and third assessments, respectively.

\section{Potential confounders: Sociodemographic and other characteristics}

All sociodemographic and other characteristics were measured at baseline, except for burden, which was measured at the three points.

Family caregiver. Sociodemographic data about the caregiver included age, gender, education level (low, middle, and higher education), and having any paid job. Caregiver burden was measured with the seven-item version of the Zarit's Burden Interview (ZBI; Zarit et al., 1980) with a Likert type-response ranging from 1 (never) to 5 (always). Total scores range from 7 to 35 and higher scores indicate a higher level of burden. Scores higher than 13 indicate clinically significant burden levels. Cronbach's alpha was 0.91 at baseline, 0.88 at the second assessment, and 0.88 at the third assessment.

Person with dementia. The time since the person with dementia was admitted to the nursing home was assessed. Furthermore, severity of the dementia was measured with the Bedford Alzheimer Nursing-Severity Scale (BANS-S; Volicer et al., 1994), which includes seven items assessing cognitive deficits, functional deficits, and frequency of pathological symptoms. The BANS-S was filled in by the nursing staff. Item scores range from 1 to 4 , and total scores range from 7 to 28 , with scores equal or higher than 17 indicating severe dementia (van der Steen et al., 2006).

\section{Statistical analyses}

Prior to the analyses, normality of the measured variables was tested. To describe the characteristics of the study sample means, standard deviations and ranges were calculated. To describe the scores obtained on the main variables, the means and standard deviations for each measurement point were calculated.

To examine the relationships between guilt feelings and the two independent variable conflicts with staff and satisfaction with care and over time, linear mixed models with random intercepts and restricted maximum likelihood estimation were used. Through mixed model analysis, an intercept and slope for each case is estimated from all available data for that participant, augmented by the data from the entire sample. Hence, although dropouts are common in longitudinal research, mixed models use all participant's available information even when dropout occurred; therefore, we did not impute missing data as unnecessary.

First, we determined whether a change in guilt feelings over time was different for the intervention and control group. Specifically, the condition (intervention vs. usual care group), time of measurement (baseline, three-months follow-up and 12 months follow-up), and the condition by time interaction were treated as fixed effects, and subjects were treated as a random effect. The result showed that the change of guilt feelings over time was not different for family caregivers in the intervention group compared with caregivers in the usual care group. We, therefore, combined the data from the intervention and usual care conditions for all further analyses; however, the condition variable was maintained in the models in order to control for its effects on the relationships we 
examined. Next, to examine the relationship between guilt and each independent variable, four models were built.

The first model involved an unadjusted model including the main independent variable (conflicts with staff or satisfaction with care), the condition (intervention vs. usual care), time of measurement (baseline, three months follow-up and 12 months follow-up), and the independent variable by time interaction treated as fixed effects, and subjects were treated as a random effect. In both cases (with conflicts with staff or satisfaction with care as independent variable), the interaction terms between the independent variable and caregivers' guilt were not significant, indicating that there were no changes in the relationship between the conflicts with staff and satisfaction with care and guilt over time, and the interaction term was, therefore, removed in the subsequent models. In the next three models, we adjusted for the potential confounders (see Measures) to examine their effect on the relationship between the outcome and independent variables.

In the first adjusted model, sociodemographic variables (gender, age, employment status, and educational level) were added as fixed effects. In the second adjusted model sociodemographic variables, time of admission, and severity of dementia were included as fixed effects. In the third adjusted model, sociodemographic variables, time of admission, the severity of dementia, and caregiver burden were entered as fixed effects. Statistical significance was considered as two-tailed $p<0.05$. Analyses were conducted in SPSS 22 and STATA 14.

\section{Results}

\section{Sample characteristics}

A total of 597 dyads of family caregivers and their relatives with dementia living in the participating nursing homes were invited to participate in the Namaste Care Family intervention study. Of the total sample, 222 did not respond to the invitation and 132 declined to participate. In addition, one dyad did not meet the inclusion criteria; three family caregivers did not return the informed consent; and eight residents with dementia died before baseline assessment, thus they and their family caregivers were unable to participate.

Finally, 231 family caregivers and their relatives with dementia residing in the participating nursing homes met the eligibility criteria and were willing to participate. Of the total sample, 222 family caregivers filled in the main study questionnaires at baseline. At the 12-month follow-up assessment, data were available for 123 participants (55.4\%). Eighty-two caregivers left the study due to the death of the resident with dementia; six dropped out because the resident was transferred to a different $\mathrm{NH}$; seven dropped out due to the resident leaving the $\mathrm{NH}$; and four refused to continue their participation in the study.

Table 1 presents the baseline characteristics of the participants. The sample of family caregivers included mainly women (71.6\%) and their mean age was 64.6 (SD 11.3) years. The family caregivers reported moderate levels of burden (M 12.9, SD 5.7), very close to the cutoff score for clinically significant burden levels (ZBI $>13)$.

There were no significant differences between those caregivers who dropped out of the study and those who did not in their baseline scores on gender $\left(\chi^{2}=0.53 ; p=0.47\right)$, age $(t=0.51 ; p=0.61)$, guilt $(t=0.11 ; p=0.91)$, conflicts with $\operatorname{staff}(t=0.08 ; p=0.94)$, satisfaction with care $(t=0.19 ; p=$ $0.25)$, and burden $(t=0.81 ; p=0.42)$. Significant differences were found in the employment status, showing that caregivers with a paid job more often dropped out compared with those without a paid job $\left(\chi^{2}=5.23: p=0.02\right)$. 
Table I. Baseline sociodemographic and clinical characteristics of participants.

\begin{tabular}{|c|c|c|}
\hline & & $n=222$ \\
\hline \multirow{2}{*}{\multicolumn{2}{|c|}{ Caregivers' age, $M(\mathrm{SD})$; caregivers' gender female, $n(\%)$}} & $64.6(11.3)$ \\
\hline & & $156(71.6 \%)$ \\
\hline \multirow[t]{3}{*}{ Caregivers' education level, $n$ (\%) } & High & $94(43.1 \%)$ \\
\hline & Medium & $113(51.8 \%)$ \\
\hline & Low & II (5.0\%) \\
\hline \multicolumn{2}{|l|}{ Caregivers with a job, $n$ (\%) } & $112(48.5 \%)$ \\
\hline \multicolumn{2}{|l|}{ Caregiver burden, $M(\mathrm{SD})$} & $12.9(5.7)$ \\
\hline \multicolumn{2}{|l|}{ Residents' age, $M(\mathrm{SD})$} & $84.7(7.5)$ \\
\hline \multicolumn{2}{|l|}{ Residents' gender female, $n(\%)$} & $167(75.2 \%)$ \\
\hline \multicolumn{2}{|c|}{ Time of admission to nursing home (years), $M(\mathrm{SD})$} & $2.8(2.6)$ \\
\hline \multicolumn{2}{|l|}{ Severity of dementia, $M$ (SD.) } & $15.0(4.5)$ \\
\hline
\end{tabular}

Note: M: mean; SD: standard deviation. Caregivers' education level low (primary education); medium (secondary education); and high (higher education). Severity of dementia = Bedford Alzheimer Nursing-Severity Scale (BANS-S). Caregiver' burden = Zarit's Burden Interview (ZBI).

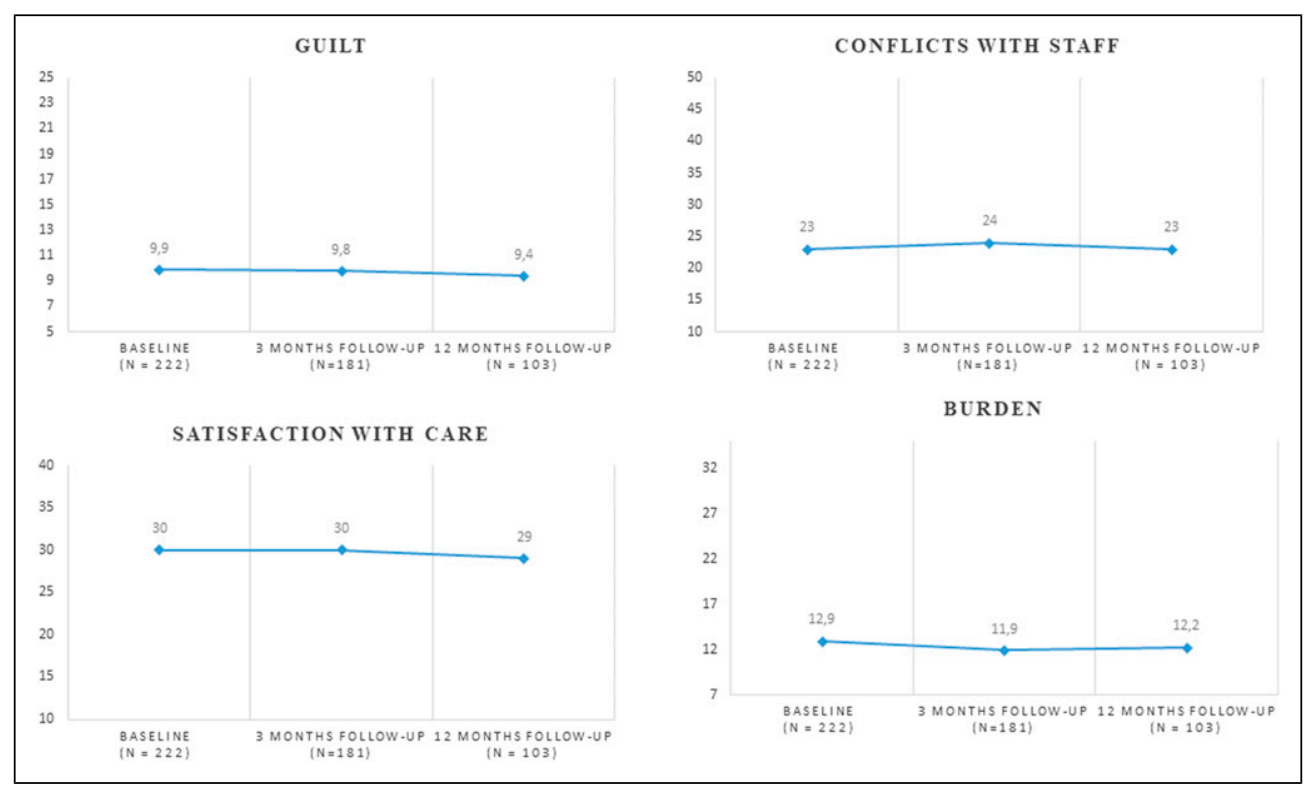

Figure I. Mean scores of main variables over time.

\section{Levels of guilt feelings, conflicts with staff, and satisfaction with care over time}

Levels of guilt feelings, frequency of conflicts with staff, and satisfaction with care and burden seemed stable over time (Figure 1). Family caregivers reported a rather low level of guilt feelings with a mean score of 9.9 (SD 4.2) at baseline and their levels were similar at the second ( $M 9.8$; SD 4.2) and third assessment ( $M 9.4$; SD 4.2). Regarding the frequency of conflicts with the staff, the caregivers reported a moderate frequency of conflicts with a mean of 22.7 (SD 7.36) at baseline, 23.5 
(SD 7.9) at the second assessment, and 23.4 (SD 7.3) at the third assessment. Family caregivers reported a mean for satisfaction with care of 29.7 (SD 4.3) at baseline, 29.5 (SD 4.3) at the second assessment, and 29.3 (SD 4.8) at the third assessment. Levels of burden were stable over time, with mean scores close to the cutoff score (13) of the ZBI.

\section{Relationship between conflicts with staff and guilt feelings}

In the unadjusted model, higher levels of conflict with staff were significantly associated with higher levels of guilt feelings $(\beta=0.11 ; p<0.001 ; 95 \% \mathrm{CI}$ : 0.06 to 0.16$)$.

Table 2 summarizes the models in which we adjusted in a stepwise fashion for socio-demographic and clinical/other characteristics which might impact the relation between conflicts with nursing home staff and caregiver's guilt levels. In the three models, adjusting for sociodemographics, clinical characteristics of the person with dementia and caregiver burden, the relationship between guilt feelings and conflicts with staff remained significant and that association was similar as the association in the unadjusted model. In the final model, conflicts and burden were the only covariates that were significantly related to guilt levels.

\section{Relationship between satisfaction with care and guilt feelings}

The unadjusted model showed that higher levels of satisfaction with care were significantly related to lower feelings of guilt over time $(\beta=-0.10 ; p<0.05 ; 95 \% \mathrm{CI}:-0.18$ to -0.01$)$.

The adjusted models are summarized in Table 3 . In the first two models, adjusted for sociodemographic and clinical characteristics of the person with dementia, that relationship remained significant. In the final model, also adjusted for caregiver burden, the relationship between satisfaction with care and guilt feelings was not significant anymore $(\beta=-0.07 ; p=0.10 ; 95 \% \mathrm{CI}:-0.16$ to 0.01 ) (see Table 3 ). In this model, only higher levels of burden were associated with higher levels of guilt over time $(\beta=0.14 ; p<0.05 ; 95 \% \mathrm{CI}: 0.06$ to 0.22$)$.

\section{Discussion}

\section{Main findings}

This study aimed to examine the relationship between family caregivers' guilt feelings and conflicts with staff and family caregivers' satisfaction with the care received by the relative with dementia living in the nursing home. Compared to previous studies, guilt feelings were rather low but stable over time. The frequency of conflicts with staff was significantly associated with higher levels of family caregivers' feelings of guilt, also after adjustment for sociodemographics, characteristics of the person with dementia, and caregiver burden. Higher levels of satisfaction with care were significantly associated with less guilt in family caregivers, but this association was not significant anymore when adjusting for caregiver burden, suggesting its important role in the explanation of guilt feelings.

\section{Interpretation and comparison with literature}

The levels of guilt reported in our study sample are lower than in previous studies carried out with the FPCR (Bramble et al., 2011; Zimmerman et al., 2013). Nevertheless, keeping in mind that the average time the relative with dementia resided in the nursing home at the time of our baseline 


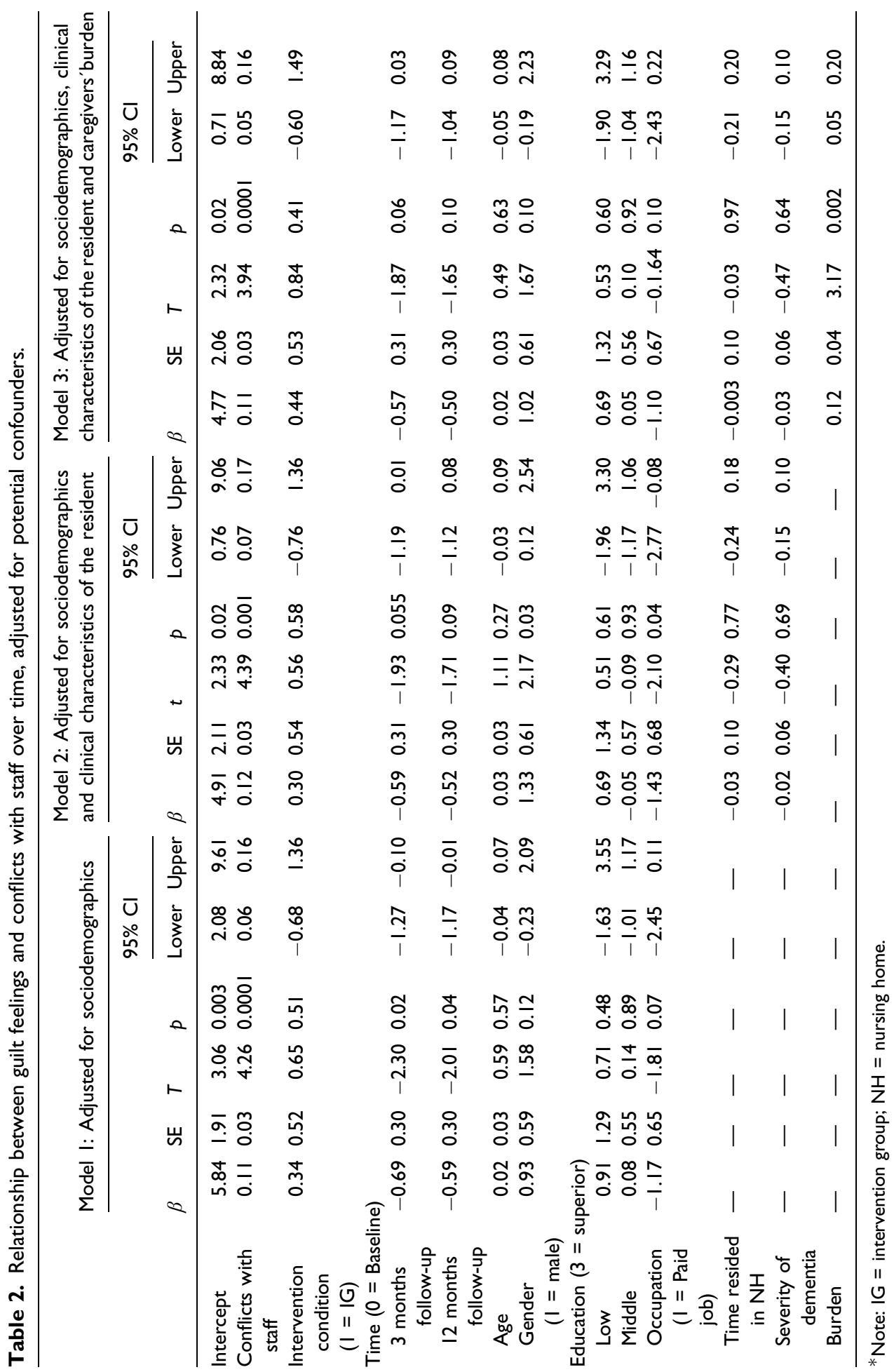




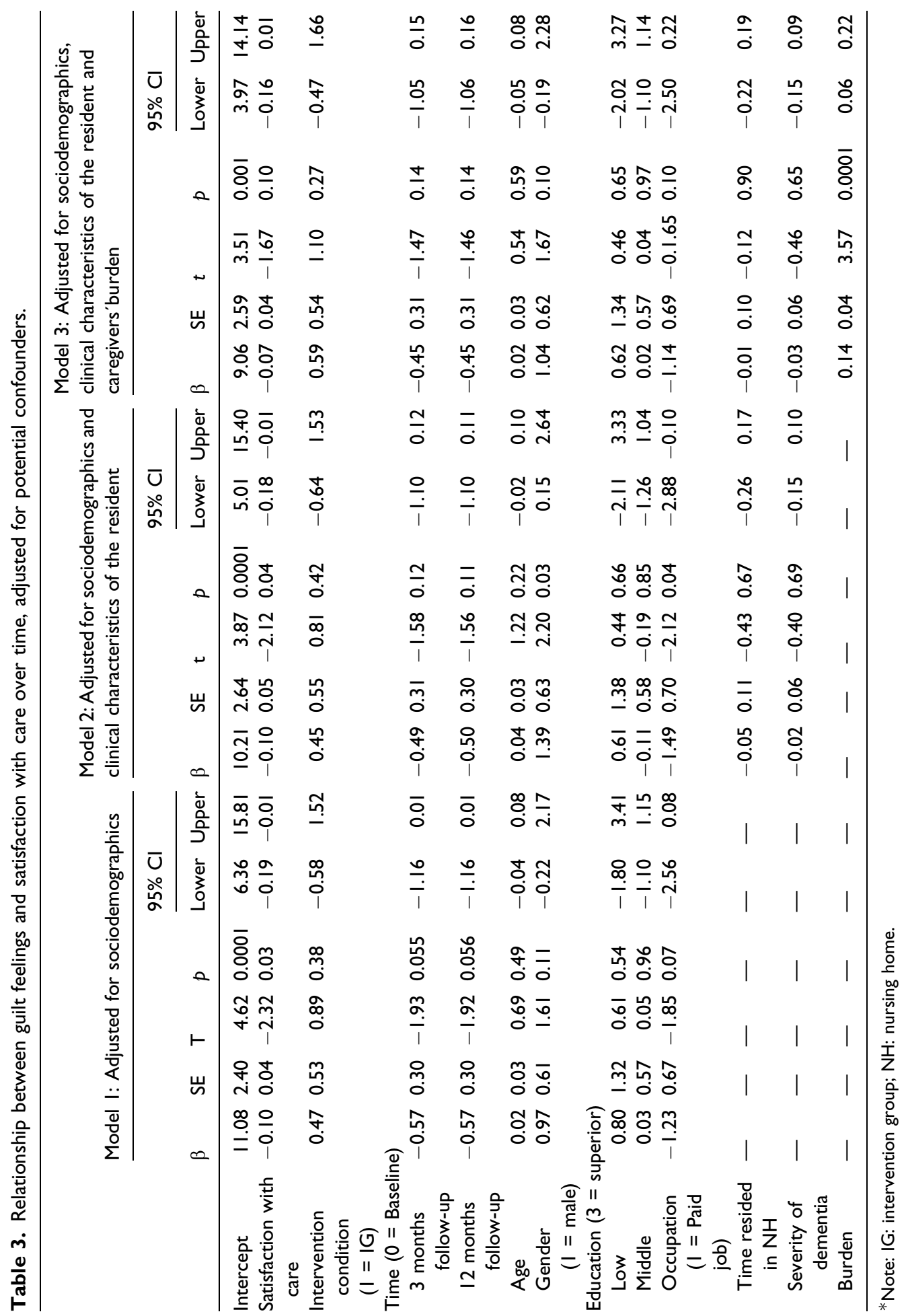


assessment was already close to three years, our results also show that guilt feelings are still experienced by family caregivers despite long-term residence. Guilt could originate from or continue several years after the nursing home admission. That finding supports previous cross-sectional literature about experiencing retained guilt feelings after admission of the person with dementia to a nursing home (Bern-Klug \& Forbes-Thompson, 2008; Church et al., 2016; Davis et al., 2019; Harstäde et al., 2013; Henningsal et al., 2013; Park, 2002; Ryan \& Scullion, 2000; Sury et al., 2013).

Potential predictors and moderating variables of guilt feelings in dementia family caregiving have been studied rarely (Losada et al., 2018), especially after admission of the person with dementia to a nursing home setting (Davis et al., 2019). The present findings show that more conflicts with the staff are associated with higher levels of guilt, and higher satisfaction with care was related to less guilt feelings. However, the relationship between satisfaction and guilt was no longer significant when adjusting for burden, indicating the relevant role of burden on the guilt feelings experienced by family caregivers.

The finding that a higher frequency of conflicts with staff is associated with more family caregivers' guilt is consistent with studies about quality of the relationships established between families and nursing home staff which have demonstrated the negative impact of dysfunctional dynamics and conflicts on caregivers' well-being (Abrahamson et al., 2009; Cohen et al., 2012; Maas et al., 2004; Pillemer et al., 2003; Roberts \& Ishler, 2017).

Regarding satisfaction with care, our sample reported lower scores than previous studies carried out with the EOLD-SWC in Dutch samples, which reported scores higher than 30 (Cohen et al., 2012; van der Steen et al., 2014; Zimmerman et al., 2015). Even so, we found that higher caregivers' satisfaction with care was associated with lower levels of guilt feelings over time. Previous crosssectional studies found that caregivers who feel more satisfied with care of the resident experience less guilt feelings (Bern-Klug \& Forbes-Thompson, 2008; Park, 2002). This finding has an important implication as it suggests that satisfaction with care may influence the emotional well-being of the family caregivers, such as their guilt feelings, and is thus very important to pay attention to. Our results suggest that guilt and perceived burden may affect satisfaction ratings, so it would be useful to include such perceptions and emotional aspects of the family caregiver in surveys aimed at assessing the families' satisfaction with professional care settings for people with dementia. Future studies should examine all of those potential mediators in satisfaction with care reports to be able to draw the right conclusions.

In addition to conflicts and satisfaction with care, our results confirm that care burden plays a relevant role in the explanation of guilt feelings experienced by family caregivers (Feast et al., 2017; Springate \& Tremont, 2014). Our study showed that higher levels of burden relate to higher feelings of guilt, showing that burden is associated with guilt in caregivers in nursing home settings, and not just with guilt experienced by caregivers of persons with dementia residing in the community (Davis et al., 2019). Furthermore, our findings suggest that burden not only has a direct effect on guilt feelings but that it also influences the relationship between conflicts with staff and satisfaction with care with guilt as, when adjusting for care burden levels in the linear models, the association between conflicts with staff and satisfaction with care variables and guilt feelings decreased. Specifically, the effect of satisfaction with care on guilt was smaller and not significant anymore when adjusted for burden. These results indicate that care burden explains a significant part of the relationship between caregiver's conflicts with staff and their satisfaction with the care and the guilt feelings experienced by caregivers, especially of satisfaction with care. These results are consistent with findings of Garity (2006), who found that higher guilt after admission was related to higher perceived burden and worse coping strategies. The association between higher burden levels in the caregiver and more guilt feelings could be explained by the family caregivers who are more 
burdened reporting more conflicts with staff and less adaptive of coping strategies to the nursing home admission and thus experience more guilt feelings than those caregivers with less perceived burden. Alternatively, the family caregivers who report more conflicts with staff may become more burdened and feel more guilty. However, with regard to the relationship between guilt and satisfaction with care, significant burden levels confound the effect of satisfaction levels on guilt feelings. The caregivers who are more burdened could employ maladaptive coping strategies (e.g., argue with the staff or cognitive beliefs about they are not doing enough) that may lead to more guilt feelings, and their satisfaction with care has less impact on their guilt than their burden levels. More studies are needed to understand the precise relationships between burden and guilt in caregivers. Future studies should examine the impact of burden on the use of different coping strategies and its consequences on caregivers' well-being (such as guilt, sadness, etc.). This may help to identify caregivers of persons with dementia at elevated risk to psychological distress.

\section{Strengths and limitations}

To our knowledge, this is the first longitudinal study examining guilt feelings and its relationship with conflicts with staff and satisfaction with care over time in family caregivers of relatives with dementia living in a nursing home. The study shows the relevant role of negative dynamics with staff and satisfaction with the care in guilt experienced during one year of follow-up.

However, the present study has various limitations. First, the sample comprises Dutch caregivers who voluntary participated in a study designed to test the effectiveness of a psychosocial intervention. That sample may not be representative of the general family caregiver population. Many caregivers could not be followed for 12 months mostly because the resident died, and a few more reasons. However, participants who completed the study and those who dropped out were similar in almost all sociodemographic characteristics and main variables of the study. Furthermore, the dropout rates were similar to other longitudinal studies carried out with nursing home residents (e.g., Vossius et al., 2018). On the other hand, the strong relationship between burden and guilt obtained should be interpreted with caution as previous studies has shown that the ZBI (Zarit et al., 1980) has items conceptually close to the guilt construct (i.e., the ZBI item "Do you feel you should be doing more for your relative" is close to the concept of guilt). In fact, some authors suggested that it contains a factor which groups guilt feelings in family caregivers (Springate \& Tremont, 2014). Furthermore, although previous studies that administered the FPCR instrument reported adequate to good reliability coefficients (Bramble et al., 2011; Zimmerman et al., 2013), the psychometric properties of the FPCR have not yet been fully examined. Also, there are other variables which were not measured in our study may be affecting the relationship between the main study variables that have been highlighted as important factors in the quality of relationships between families and nursing home staff (Garity, 2006; Pillemer et al., 2003), such as coping strategies or communication skills of the caregiver. It would be interesting if future studies analyze the effect of these variables on caregivers' guilt. Also, the satisfaction with the care could depend on several factors of the nursing home (i.e., availability of resources, cleanliness of installations...), so futures studies could control for and examine the impact of the nursing homes' characteristics on satisfaction and guilt experienced by the families.

Finally, alternative explanations can be given to the findings. For example, the caregivers' guilt levels may act as triggers of conflicts with staff. In this line, Caputo (2021) showed that guilt feelings related to feelings of uncertainty about the effectiveness of the care received by the relative with dementia, especially in those caregivers who experience more burden. Hence, caregivers who experience higher levels of guilt may interact with the staff of the nursing home in order to ensure 
that their relative obtains the preferred care and alleviate these guilt feelings. However, the probability that a conflict emerges with the professionals when the caregiver expresses their dissatisfaction with the care offered would be higher for caregivers who are experiencing negative feelings, burden, or present poorer communication skills. Future studies are needed to confirm our results.

\section{Implications and conclusion}

In sum, guilt feelings are experienced by family caregivers of nursing home residents with dementia, also in the years after admission, and these feelings are stable over time. The present study has shown that, despite the presence of caregiver burden, more or a higher level of conflicts with nursing home staff is significantly associated with higher levels of guilt experienced by the caregiver. Techniques and interventions based on Acceptance and Commitment Therapy (ACT; Hayes et al., 1999) can be an interesting therapeutic strategy for addressing guilt feelings, as therapists and nurses can alleviate family caregivers' guilt through exercises aimed at normalizing, validating, and accepting the presence of guilt during the caregiving experience. ACT-based interventions have demonstrated to be effective in reducing depressive, anxious, and guilt feelings in dementia family caregivers (Gallego-Alberto et al., 2019; Losada et al., 2015).

\section{Declaration of conflicting interests}

The author(s) declared no potential conflicts of interest with respect to the research, authorship, and/or publication of this article.

\section{Funding}

The author(s) disclosed receipt of the following financial support for the research, authorship, and/or publication of this article: Fonds NutsOhra (Grand No. 1405181), University Network of the Care sector South Holland, and ZonMw (Grand No. 733050302).

\section{ORCID iDs}

Laura Gallego-Alberto (D) https://orcid.org/0000-0002-0094-834X

Hanneke JA Smaling (D) https://orcid.org/0000-0002-7836-431X

\section{References}

Abrahamson, K., Jill Suitor, J., \& Pillemer, K. (2009). Conflict between nursing home staff and residents' families: Does it increase burnout? Journal of Aging and Health, 21(6), 895-912. DOI: 10.1177/ 0898264309340695.

Bern-Klug, M., \& Forbes-Thompson, S. (2008). Family members' responsibilities to nursing home residents: "She is the only mother I got". Journal of Gerontological Nursing, 34(2), 43-52. DOI: 10.3928/0098913420080201-02.

Bramble, M., Moyle, W., \& Shum, D. (2011). A quasi-experimental design trial exploring the effect of a partnership intervention on family and staff well-being in long-term dementia care. Aging \& Mental Health, 15(8), 995-1007. DOI: 10.1080/13607863.2011.583625.

Caputo, A. (2021). The emotional experience of caregiving in dementia: Feelings of guilt and ambivalence underlying narratives of family caregivers. Dementia, 1471301221989604, 147130122198960. DOI: 10. $1177 / 1471301221989604$. 
Church, L. L., Schumacher, K. L., \& Thompson, S. A. (2015). Mixed-methods exploration of family caregiver strain in the nursing home. Journal of Hospice \& Palliative Nursing, 18(1), 46-52. DOI: 10.1097/NJH. 0000000000000209.

Cohen, L. W., van der Steen, J. T., Reed, D., Hodgkinson, J. C., van Soest-Poortvliet, M. C., Sloane, P. D., \& Zimmerman, S. (2012). Family perceptions of end-of-life care for long-term care residents with dementia: Differences between the United States and the The Netherlands. Journal of the American Geriatrics Society, 60(2), 316-322. DOI: 10.1111/j.1532-5415.2011.03816.x.

Cronfalk, B. S., Ternestedt, B. M., \& Norberg, A. (2017). Being a close family member of a person with dementia living in a nursing home. Journal of Clinical Nursing, 26(21-22), 3519-3528.

Davis, J. D., Hill, B. D., Pillemer, S., Taylor, J., \& Tremont, G. (2019). Guilt after placement questionnaire: A new instrument to assess caregiver emotional functioning following nursing home placement. Aging \& Mental Health, 23(3), 352-356. DOI: 10.1080/13607863.2017.1423029.

Feast, A., Orrell, M., Russell, I., Charlesworth, G., \& Moniz-Cook, E. (2017). The contribution of caregiver psychosocial factors to distress associated with behavioural and psychological symptoms in dementia. International Journal of Geriatric Psychiatry, 32(1), 76-85. DOI: 10.1002/gps.4447.

Gallego-Alberto, L., Losada, A., Vara, C., Olazarán, J., Muñiz, R., \& Pillemer, K. (2018). Psychosocial predictors of anxiety in nursing home staff. Clinical Gerontologist, 41(4), 282-292. DOI: 10.1080/ 07317115.2017.1370056.

Gallego-Alberto, L., Márquez-González, M., Romero-Moreno, R., Cabrera, I., \& Losada, A. (2019). Pilot study of a psychotherapeutic intervention for reducing guilt feelings in highly distressed dementia family caregivers (Innovative practice). Dementia, 20, 759, 769. DOI: 10.1177/1471301219886761.

Garity, J. (2006). Caring for a family member with Alzheimer's disease: Coping with caregiver burden postnursing home placement. Journal of Gerontological Nursing, 32(6), 39-48. DOI: 10.3928/0098913420060601-07.

Gaugler, J. E., Mittelman, M. S., Hepburn, K., \& Newcomer, R. (2009). Predictors of change in caregiver burden and depressive symptoms following nursing home admission. Psychology and Aging, 24(2), 385, 396. DOI: $10.1037 / \mathrm{a} 0016052$.

Gaugler, J. E., Pot, A. M., \& Zarit, S. H. (2007). Long-term adaptation to institutionalization in dementia caregivers. The Gerontologist, 47(6), 730-740. DOI: 10.1093/geront/47.6.730.

Givens, J. L., Lopez, R. P., Mazor, K. M., \& Mitchell, S. L. (2012). Sources of stress for family members of nursing home residents with advanced dementia. Alzheimer Disease and Associated Disorders, 26(3), 254-259. DOI: 10.1097/WAD.0b013e31823899e4.

Gladstone, J. W., Dupuis, S. L., \& Wexler, E. (2006). Changes in family involvement following a relative's move to a long-term care facility. Canadian Journal on Aging/La Revue canadienne du vieillissement, 25(1), 93-106. DOI: $10.1353 /$ cja.2006.0022.

Graneheim, U. H., Johansson, A., \& Lindgren, B.-M. (2014). Family caregivers' experiences of relinquishing the care of a person with dementia to a nursing home: insights from a meta-ethnographic study. Scandinavian Journal of Caring Sciences, 28(2), 215-224. DOI: 10.1111/scs.12046.

Haesler, E., Bauer, M., \& Nay, R. (2007). Staff-family relationships in the care of older people: A report on a systematic review. Research in Nursing \& Health, 30(4), 385-398. DOI: 10.1002/nur.20200.

Harrop, E., Morgan, F., Byrne, A., \& Nelson, A. (2016). "It still haunts me whether we did the right thing": A qualitative analysis of free text survey data on the bereavement experiences and support needs of family caregivers. BMC Palliative Care, 15(1), 92. DOI: 10.1186/s12904-016-0165-9.

Harstäde, C. W., Andershed, B., Roxberg, А., \& Brunt, D. (2013). Feelings of guilt-experiences of next of kin in end-of-life care. Journal of Hospice \& Palliative Nursing, 15(1), 33-40. DOI: 10.1097/NJH. 0b013e318262332c.

Hayes, S. C., Strosahl, K., \& Wilson, K. G. (1999). Acceptance and commitment therapy: Understanding and treating human suffering. Guilford.

Hennings, J., Froggatt, K., \& Payne, S. (2013). Spouse caregivers of people with advanced dementia in nursing homes: A longitudinal narrative study. Palliative Medicine, 27(7), 683-691. DOI: 10.1177/ 0269216313479685 . 
Høgsnes, L., Melin-Johansson, C., Norbergh, K. G., \& Danielson, E. (2014). The existential life situations of spouses of persons with dementia before and after relocating to a nursing home. Aging \& Mental Health, 18(2), 152-160. DOI: 10.1080/13607863.2013.818100.

Houttekier, D., Cohen, J., Bilsen, J., Addington-Hall, J., Onwuteaka-Philipsen, B. D., \& Deliens, L. (2010). Place of death of older persons with dementia. A study in five European countries. Journal of the American Geriatrics Society, 58(4), 751-756. DOI: 10.1111/j.1532-5415.2010.02771.x.

Kugler, K., \& Jones, W. H. (1992). On conceptualizing and assessing guilt. Journal of Personality and Social Psychology, 62(2), 318, 327. DOI: 10.1037/0022-3514.62.2.318.

Losada, A., Márquez-González, M., Romero-Moreno, R., Mausbach, B. T., López, J., Fernández-Fernández, V., \& Nogales-González, C. (2015). Cognitive-behavioral therapy (CBT) versus acceptance and commitment therapy (ACT) for dementia family caregivers with significant depressive symptoms: Results of a randomized clinical trial. Journal of Consulting and Clinical Psychology, 83(4), 760-772. DOI: 10.1037/ ccp0000028.

Losada, A., Márquez-González, M., Vara-García, C., Gallego-Alberto, L., Romero-Moreno, R., \& Pillemer, K. (2018). Ambivalence and guilt feelings: Two relevant variables for understanding caregivers' depressive symptomatology. Clinical Psychology \& Psychotherapy, 25(1), 59-64. DOI: 10.1002/cpp.2116.

Maas, M. L., Reed, D., Park, M., Specht, J. P., Schutte, D., Kelley, L. S., Swanson, E. A., Trip-Reimer, T.-T., \& Buckwalte K, . C. (2004). Outcomes of family involvement in care intervention for caregivers of individuals with dementia. Nursing Research, 53(2), 76-86. DOI: 10.1097/00006199-200403000-00003.

Ma, M., Dorstyn, D., Ward, L., \& Prentice, S. (2018). Alzheimers' disease and caregiving: A meta-analytic review comparing the mental health of primary carers to controls. Aging \& Mental Health, 22(11), 1395-1405. DOI: 10.1080/13607863.2017.1370689.

Nikzad-Terhune, K. A., Anderson, K. A., Newcomer, R., \& Gaugler, J. E. (2010). Do trajectories of at-home dementia caregiving account for burden after nursing home placement? A growth curve analysis. Social Work in Health Care, 49(8), 734-752. DOI: 10.1080/00981381003635296.

Park, M. (2002). Korean family caregivers' perceptions of care in dementia care units. Journal of Korean Academy of Nursing, 32(7), 967-976.

Pillemer, K., Suitor, J. J., Henderson Jr, C. R., Meador, R., Schultz, L., Robison, J., \& Hegeman, C. (2003). A cooperative communication intervention for nursing home staff and family members of residents. The Gerontologist, 43(2), 96-106. DOI:10.1093/geront/43.suppl_2.96.

Pinquart, M., \& Sörensen, S. (2003). Differences between caregivers and noncaregivers in psychological health and physical health: A meta-analysis. Psychology and aging, 18(2), 250-267.

Roberts, A. R., \& Ishler, K. J. (2017). Family involvement in the nursing home and perceived resident quality of life. The Gerontologist, 58(6), 1033-1043. DOI: 10.1093/geront/gnx108.

Ryan, A. A., \& Scullion, H. F. (2000). Nursing home placement: An exploration of the experiences of family carers. Journal of Advanced Nursing, 32(5), 1187-1195. DOI: 10.1046/j.1365-2648.2000.01589.x.

Smaling, H. J., Joling, K. J., Van de Ven, P. M., Bosmans, J. E., Simard, J., Volicer, L., Achterberg, W. P., Francke, A. L., \& van der Steen, J. T. (2018). Effects of the Namaste Care Family Programme on quality of life of nursing home residents with advanced dementia and on family caregiving experiences: Study protocol of a cluster-randomised controlled trial. BMJ Open, 8(10), e025411. DOI: 10.1136/bmjopen-2018-025411.

Springate, B. A., \& Tremont, G. (2014). Dimensions of caregiver burden in dementia: Impact of demographic, mood, and care recipient variables. The American Journal of Geriatric Psychiatry, 22(3), 294-300. DOI: 10. 1016/j.jagp.2012.09.006.

Sury, L, Burns, K., \& Brodaty, H. (2013). Moving in: Adjustment of people living with dementia going into a nursing home and their families. International Psychogeriatrics, 25(6), 867-876. DOI: $10.1017 /$ S1041610213000057.

Toot, S., Swinson, T., Devine, M., Challis, D., \& Orrell, M. (2017). Causes of nursing home placement for older people with dementia: A systematic review and meta-analysis. International Psychogeriatrics, 29(2), 195-208. DOI: 10.1017/S1041610216001654. 
van der Steen, J. T., Gijsberts, M.-J. H., Hertogh, C. M., \& Deliens, L. (2014). Predictors of spiritual care provision for patients with dementia at the end of life as perceived by physicians: A prospective study. $B M C$ Palliative Care, 13(1), 61. DOI: 10.1186/1472-684X-13-61.

van der Steen, J.T., Volicer, L., Gerritsen, D.L., Kruse, R.L., Ribbe, M.W., \& Mehr, D.R. (2006). Defining severe dementia with the minimum data set. International Journal of Geriatric Psychiatry, 21(11), 1099-1106. DOI: 10.1002/gps.1618.

Volicer, L., Hurley, A. C., \& Blasi, Z. V. (2001). Scales for evaluation of end-of-life care in dementia. Alzheimer Disease and Associated Disorders, 15(4), 194-200. DOI: 10.1097/00002093-200110000-00005.

Volicer, L., Hurley, A. C., Lathi, D. C., \& Kowall, N. W. (1994). Measurement of severity in advanced Alzheimer's disease. Journal of gerontology, 49(5), 223-226. DOI: 10.1093/geronj/49.5.M223.

Vossius, C., Selbæk, G., Šaltyte Benth, J., \& Bergh, S. (2018). Mortality in nursing home residents: A longitudinal study over three years. Plos One, 13(9), e0203480. DOI: 10.1371/journal.pone.0203480.

Zarit, S. H., Reever, K. E., \& Bach-Peterson, J. (1980). Relatives of the impaired elderly: Correlates of feelings of burden. The Gerontologist, 20(6), 649-655. DOI: 10.1093/geront/20.6.649.

Zimmerman, S., Cohen, L. W., Reed, D., Gwyther, L. P., Washington, T., Cagle, J. G., Sloane, P. D., \& Preisser, J. S. (2013). Families matter in long-term care: results of a group-randomized trial. Seniors Housing \& Care Journal, 21(1), 3-20.

Zimmerman, S., Cohen, L., van der Steen, J. T., Reed, D., van Soest-Poortvliet, M. C., Hanson, L. C., \& Sloane, P. D. (2015). Measuring end-of-life care and outcomes in residential care/assisted living and nursing homes. Journal of Pain and Symptom Management, 49(4), 666-679. DOI: 10.1016/j.jpainsymman.2014.08.009.

Laura Gallego-Alberto: Assistant Professor at the Universidad Autónoma de Madrid, Spain. Her research is focused on analyzing the guilt experienced by informal caregivers of people with dementia and its effects on caregivers' psychological and physical health, and the design and implementation of psychological interventions aimed at reducing the distress of caregivers.

Hanneke JA Smaling: Senior Researcher at Leiden University Medical Center, The Netherlands. Her research is focus in analyzing variables related to the care of dementia and palliative care patient and implementing psychosocial interventions aimed at improving the quality of life of those patients.

Anneke L Francke: Chair in "Nursing at-the-end-of-life", by NIVEL (Netherlands institute of health services research). Her research interest are focused on nursing, palliative care and dementia care. She wrote numerous articles within the fields of nursing and end-of-life-care.

Tim van de Brug: Research associate at VU University Medical Center, Amsterdam, The Netherlands. His research is focused on high-dimensional statistics and machine learning for the analysis of medical imaging data.

Jenny T van der Steen: Epidemiologist and Associate professor at Leiden University Medical Center. She is working in the field of end-of-life care, and in particular palliative care for nursing home residents with dementia. She is the lead author of the European Association for Palliative Care (EAPC) white paper on palliative care in dementia.

Karlijn J Joling: Senior researcher at the Department of General practice and Elderly care medicine of the VU, University Medical Center. Her research interests are focused on a) dementia care and variables related to informal caregivers' mental health; b) on designing psychological interventions for prevent distress in this population. 